\title{
Oscillation of nonlinear third-order difference equations with mixed neutral terms
}

\author{
Jehad Alzabut ${ }^{*}$ (D), Martin Bohner ${ }^{2}$ and Said R. Grace ${ }^{3}$
}

\section{"Correspondence:}

jalzabut@psu.edu.sa

'Department of Mathematics and

General Sciences, Prince Sultan

University, 11586 Riyadh, Saudi

Arabia

Full list of author information is

available at the end of the article

\begin{abstract}
In this paper, new oscillation results for nonlinear third-order difference equations with mixed neutral terms are established. Unlike previously used techniques, which often were based on Riccati transformation and involve limsup or liminf conditions for the oscillation, the main results are obtained by means of a new approach, which is based on a comparison technique. Our new results extend, simplify, and improve existing results in the literature. Two examples with specific values of parameters are offered.
\end{abstract}

MSC: $34 \mathrm{~N} 05 ; 39 \mathrm{~A} 10$

Keywords: Oscillation; Comparison; Nonlinear third-order difference equations; Mixed neutral terms

\section{Introduction and preliminaries}

Oscillation of solutions for third-order difference equations has received comparably little attention, although such equations are of importance in many fields of science such as economics, physics, mathematical biology, and other areas of mathematics $[3,4,6,7,10,11$, $13,14,24,27,31,32,35-37]$. It is worth to mention that third-order difference equations may have totally different behavior from corresponding third-order differential equations; see the explicit example in [9]. On the other hand, oscillation of solutions for difference equations of first and second order have been extensively investigated in the literature; see the monographs $[1,2,8]$ and the papers $[5,12,15-19,21,23,25,26,30,33]$.

In this study, we consider a nonlinear third-order difference equation with mixed nonlinear neutral terms. We obtain conditions guaranteeing oscillation of solutions of this equation. The main results are proved by using a comparison technique with first-order equations. Such an approach was effectively used for other types of equations in [20, 22]. To demonstrate this, we present two examples, which cannot be discussed using any of the previously established results.

We consider the equation

$$
\Delta\left(p_{1}(t)\left(\Delta^{2} y(t)\right)^{\alpha_{1}}\right)=p_{2}(t) x^{\alpha_{2}}(t-m+1)+p_{3}(t) x^{\alpha_{3}}\left(t+m^{*}\right),
$$

(c) The Author(s) 2021. This article is licensed under a Creative Commons Attribution 4.0 International License, which permits use sharing, adaptation, distribution and reproduction in any medium or format, as long as you give appropriate credit to the original author(s) and the source, provide a link to the Creative Commons licence, and indicate if changes were made. The images or other third party material in this article are included in the article's Creative Commons licence, unless indicated otherwise in a credit line to the material. If material is not included in the article's Creative Commons licence and your intended use is not permitted by statutory regulation or exceeds the permitted use, you will need to obtain permission directly from the copyright holder. To view a copy of this licence, visit http://creativecommons.org/licenses/by/4.0/. 
where $y(t)=x(t)+p_{4}(t) x^{\alpha_{4}}(t-k)-p_{5}(t) x^{\alpha_{5}}(t-k)$, and subject to the assumptions:

(i) $\alpha_{1}, \alpha_{2}, \alpha_{3}, \alpha_{4}, \alpha_{5}$ are the ratios of positive odd integers, $\alpha_{1} \geq 1$,

(ii) $p_{1}, p_{2}, p_{3}, p_{4}, p_{5}: \mathbb{Z} \rightarrow(0, \infty)$ are sequences,

(iii) $m, m^{*}, k \in \mathbb{N}$ are such that $m>2, m^{*}>2, k<m-1$.

A solution of (1.1) is called oscillatory if it is neither eventually negative nor eventually positive. We call (1.1) oscillatory provided that all its solutions are oscillatory.

The objective of this paper is to offer conditions ensuring oscillation of (1.1) whenever

$$
\alpha_{4}<1<\alpha_{5} \quad \text { or } \quad \alpha_{4}<\alpha_{5} \leq 1
$$

and subject to the assumption

$$
P_{1}\left(t, t_{1}\right) \rightarrow \infty \quad \text { as } t \rightarrow \infty, \quad \text { where } P_{1}(v, u):=\sum_{\tau=u}^{v-1} \frac{1}{p_{1} \frac{1}{\alpha_{1}}(\tau)} .
$$

In view of the results established in the literature and to the best of our observations, there are no oscillation results for (1.1).

This paper is organized as follows: In Sect. 2, we give some auxiliary results and introduce some notation. Sect. 3 features the main results of the paper. We present our investigations under two cases for (1.1). The first case is when $\alpha_{4}<1<\alpha_{5}$, and the other case is when $\alpha_{4}<\alpha_{5} \leq 1$. Our approach is based on a comparison technique with first-order difference equations. In Sect. 4, two examples are provided in order to illustrate our main theorems.

\section{Auxiliary results and notations}

We start with the following fundamental result. See [22, Lemma 1], and for the proof of (I), see [29, Lemma 2.2].

\section{Lemma 2.1}

(I) If the first-order delay difference inequality

$$
\Delta y(t)+p_{2}(t) y^{\gamma}(t-m+1) \leq 0
$$

has an eventually positive solution, then so does the corresponding delay difference equation.

(II) If the first-order advanced difference inequality

$$
\Delta y(t)-p_{2}(t) y^{\gamma}\left(t+m^{*}\right) \geq 0
$$

has an eventually positive solution, then so does the corresponding advanced difference equation.

We also need the following lemmas.

Lemma 2.2 (see [28]) If $X, Y \geq 0$, then

$$
X^{\lambda}+(\lambda-1) Y^{\lambda}-\lambda X Y^{\lambda-1} \geq 0 \text { for } \lambda>1
$$


and

$$
X^{\lambda}-(1-\lambda) Y^{\lambda}-\lambda X Y^{\lambda-1} \leq 0 \quad \text { for } 0<\lambda<1 \text {. }
$$

Lemma 2.3 Assume (1.2). Then

$\Delta Y(t)>0 \quad$ eventually, where $Y:=p_{1}\left(\Delta^{2} y\right)^{\alpha_{1}}$

implies that eventually one of the following four situations occur:

Case PPP. $\quad y(t)>0, \quad \Delta y(t)>0, \quad \Delta^{2} y(t)>0$;

Case PPN. $\quad y(t)>0, \quad \Delta y(t)>0, \quad \Delta^{2} y(t)<0$;

Case NNN. $\quad y(t)<0, \quad \Delta y(t)<0, \quad \Delta^{2} y(t)<0$;

Case NPN. $\quad y(t)<0, \quad \Delta y(t)>0, \quad \Delta^{2} y(t)<0$.

Proof By (2.3), there exists $t_{0} \in \mathbb{N}_{0}$ satisfying

$\Delta Y(t)>0 \quad$ for all $t \geq t_{0}$.

We first assume that

$$
\text { there exists } t_{1} \geq t_{0} \quad \text { with } Y\left(t_{1}\right)>0 \text {. }
$$

Then, for all $t \geq t_{1}$, we have

$$
Y(t)=Y\left(t_{1}\right)+\sum_{\tau=t_{1}}^{t-1} \Delta Y(\tau) \stackrel{(2.4)}{\geq} Y\left(t_{1}\right) \stackrel{(2.5)}{>} 0 .
$$

Hence,

$$
\Delta^{2} y(t)>0 \quad \text { for all } t \geq t_{1} \text {. }
$$

Now, for $t \geq t_{1}$, we get

$$
\begin{aligned}
\Delta y(t) & =\Delta y\left(t_{1}\right)+\sum_{\tau=t_{1}}^{t-1} \Delta^{2} y(\tau)=\Delta y\left(t_{1}\right)+\sum_{\tau=t_{1}}^{t-1} \frac{Y^{\frac{1}{\alpha_{1}}}(\tau)}{p_{1}^{\frac{1}{\alpha_{1}}}(\tau)} \\
& \stackrel{(2.4)}{\geq} \Delta y\left(t_{1}\right)+\sum_{\tau=t_{1}}^{t-1} \frac{Y^{\frac{1}{\alpha_{1}}}\left(t_{1}\right)}{p_{1}^{\frac{1}{\alpha_{1}}}\left(t_{1}\right)}=\Delta y\left(t_{1}\right)+Y^{\frac{1}{\alpha_{1}}}\left(t_{1}\right) P_{1}\left(t, t_{1}\right) \stackrel{(2.5)}{\rightarrow} \infty \quad \text { as } t \rightarrow \infty,
\end{aligned}
$$

due to (1.2). Thus,

$$
\text { there exists } t_{2} \geq t_{1} \quad \text { with } \Delta y(t)>0 \quad \text { for all } t \geq t_{2} \text {. }
$$


Hence, for $t \geq t_{2}$, we obtain

$$
\begin{aligned}
y(t) & =y\left(t_{2}\right)+\sum_{\tau=t_{2}}^{t-1} \Delta y(\tau) \stackrel{(2.6)}{\geq} y\left(t_{2}\right)+\sum_{\tau=t_{2}}^{t-1} \Delta y\left(t_{2}\right) \\
& =y\left(t_{2}\right)+\left(t-\tau_{2}\right) \Delta y\left(t_{2}\right) \stackrel{(2.7)}{\rightarrow} \infty \quad \text { as } t \rightarrow \infty .
\end{aligned}
$$

Therefore,

$$
\text { there exists } t_{3} \geq t_{2} \quad \text { with } y(t)>0 \quad \text { for all } t \geq t_{3} \text {. }
$$

By (2.6), (2.7), and (2.8), we have

$$
y(t)>0, \quad \Delta y(t)>0, \quad \Delta^{2} y(t)>0 \quad \text { for all } t \geq t_{3},
$$

so Case PPP holds. Next, if (2.5) does not hold, then the only other possibility is

$$
Y(t)<0 \quad \text { for all } t \geq t_{0}
$$

and hence

$$
\Delta^{2} y(t)<0 \quad \text { for all } t \geq t_{0} \text {. }
$$

We assume that

$$
\text { there exists } t_{1} \geq t_{0} \quad \text { with } \Delta y\left(t_{1}\right)<0 \text {. }
$$

Then, for all $t \geq t_{1}$, we have

$$
\Delta y(t)=\Delta y\left(t_{1}\right)+\sum_{\tau=t_{1}}^{t-1} \Delta^{2} y(\tau) \stackrel{(2.9)}{\leq} \Delta y\left(t_{1}\right) \stackrel{(2.10)}{<} 0 .
$$

Thus,

$$
\Delta y(t)<0 \quad \text { for all } t \geq t_{1} \text {. }
$$

Now, for $t \geq t_{1}$, we get

$$
\begin{aligned}
y(t) & =y\left(t_{1}\right)+\sum_{\tau=t_{1}}^{t-1} \Delta y(\tau) \stackrel{(2.9)}{\leq} y\left(t_{1}\right)+\sum_{\tau=t_{1}}^{t-1} \Delta y\left(t_{1}\right) \\
& =y\left(t_{1}\right)+\left(t-t_{1}\right) \Delta y\left(t_{1}\right) \stackrel{(2.10)}{\rightarrow}-\infty \quad \text { as } t \rightarrow \infty .
\end{aligned}
$$

Hence,

$$
\text { there exists } t_{2} \geq t_{1} \quad \text { with } y(t)<0 \quad \text { for all } t \geq t_{2} \text {. }
$$


By (2.9), (2.11), and (2.12), we have

$$
y(t)<0, \quad \Delta y(t)<0, \quad \Delta^{2} y(t)<0 \quad \text { for all } t \geq t_{2},
$$

so Case NNN holds. Next, if (2.10) does not hold, then the only other possibility is

$$
\Delta y(t)>0 \text { for all } t \geq t_{0}
$$

We assume that

$$
\text { there exists } t_{1} \geq t_{0} \quad \text { with } y\left(t_{1}\right)>0 \text {. }
$$

Then, for all $t \geq t_{1}$, we have

$$
y(t)=y\left(t_{1}\right)+\sum_{\tau=t_{1}}^{t-1} \Delta y(\tau) \stackrel{(2.13)}{\geq} y\left(t_{1}\right) \stackrel{(2.14)}{>} 0 \quad \text { for all } t \geq t_{1} .
$$

By (2.9), (2.13), and (2.15), we have

$$
y(t)>0, \quad \Delta y(t)>0, \quad \Delta^{2} y(t)<0 \quad \text { for all } t \geq t_{1},
$$

so Case PPN holds. Finally, if (2.14) does not hold, then the only other possibility is

$$
y(t)<0 \quad \text { for all } t \geq t_{0}
$$

By (2.9), (2.13), and (2.16), we have

$$
y(t)<0, \quad \Delta y(t)>0, \quad \Delta^{2} y(t)<0 \quad \text { for all } t \geq t_{1},
$$

so Case NPN holds. There are no other cases. See Table 1 for an illustration of the proof.

\begin{tabular}{|c|c|c|c|c|c|}
\hline & $\Delta^{2} y(t)$ & & $\Delta y(t)$ & & $y(t)$ \\
\hline (2.5) & P & & P & & P \\
\hline \multirow[t]{3}{*}{ (2.9) } & $\mathrm{N}$ & $(2.10)$ & $\mathrm{N}$ & & $\mathrm{N}$ \\
\hline & & $(2.13)$ & $P$ & $(2.14)$ & $P$ \\
\hline & & & & $(2.16)$ & $\mathrm{N}$ \\
\hline
\end{tabular}

Throughout the remainder of the paper, we suppose that

$$
k_{0}, k_{1}, k_{2}, k_{3} \in \mathbb{N} \quad \text { satisfy } 2 k_{0}<m^{*}, k_{1}<m+1 \text {, and } k_{2}<k_{3}<m+1-k \text {. }
$$

For convenience, we introduce the notations

$$
\xi_{0}(t):=t+m^{*}-2 k_{0}, \quad \xi_{1}(t):=t-m+k_{1}-1, \quad \xi_{2}(t):=t-m+k-1,
$$

Table 1 Illustration of the proof of Lemma 2.3 


$$
\begin{aligned}
& \xi_{3}(t):=\xi_{2}(t)+k_{3}, \quad \Lambda_{0}(t):=\sum_{\tau=t-k_{0}}^{t-1}\left(\frac{1}{p_{1}(\tau)} \sum_{s=\tau-k_{0}}^{\tau-1} p_{3}(s)\right)^{\frac{1}{\alpha_{1}}}, \\
& \Lambda_{1}(t):=p_{2}(t)\left((t-m+1) P_{1}\left(t-m+k_{1}, t-m\right)\right)^{\alpha_{2}}, \\
& \Lambda_{2}(t):=Q(t)\left(\sum_{\tau=t_{1}}^{t-m+k} P_{1}\left(\tau, t_{1}\right)\right)^{\frac{\alpha_{2}}{\alpha_{5}}}, \\
& \Lambda_{3}(t):=k_{2}^{\frac{\alpha_{2}}{\alpha_{5}}} Q(t) P_{1} \frac{\alpha_{2}}{\alpha_{5}}\left(t-m+k+k_{3}, t-m+k+k_{2}\right), \quad Q(t):=\frac{p_{2}(t)}{p_{5} \frac{\alpha_{2}}{\alpha_{5}}(t-m+k+1)} .
\end{aligned}
$$

\section{Remark 2.4}

1. Note that, due to the assumptions $m>2, m^{*}>2$, and $k<m-1$, it is always possible to find $k_{0}, k_{1}, k_{2}, k_{3}$ such that (2.17) holds, e.g., one may pick

$$
k_{0}=k_{1}=k_{2}=1 \quad \text { and } \quad k_{3}=2
$$

2. Note that $\xi_{0}(t)>t$ holds always since $m^{*}-2 k_{0}>0$. Hence, equations involving $\xi_{0}$ are of advanced type. Moreover, $\xi_{1}(t)<t, \xi_{2}(t)<t$, and $\xi_{3}(t)<t$ always since $m+1-k_{1}>0$, $m+1-k>0$, and $m+1-k-k_{3}>0$. Hence, equations involving $\xi_{1}, \xi_{2}, \xi_{3}$ are of delay type.

\section{Main results}

Now we present our first oscillation result.

Theorem 3.1 Let $\alpha_{4}<1<\alpha_{5}$. Suppose that (i)-(iii), (1.2), and (2.17) hold. Assume that there exists $p: \mathbb{Z} \rightarrow(0, \infty)$ such that

$$
\begin{gathered}
\lim _{t \rightarrow \infty}\left(g_{1}(t)+g_{2}(t)\right)=0, \quad \text { where } \\
g_{1}(t):=\left(1-\alpha_{4}\right) \alpha_{4}{ }^{\frac{\alpha_{4}}{1-\alpha_{4}}} p^{\frac{\alpha_{4}}{\alpha_{4}-1}(t) p_{4}{ }^{\frac{1}{1-\alpha_{4}}}(t) \text { and }} \\
g_{2}(t):=\left(\alpha_{5}-1\right) \alpha_{5}{ }^{\frac{\alpha_{5}}{1-\alpha_{5}}} p^{\frac{\alpha_{5}}{\alpha_{5}-1}}(t) p_{5}{ }^{\frac{1}{1-\alpha_{5}}}(t) .
\end{gathered}
$$

Let $\theta_{0}, \theta_{1} \in(0,1)$. If the first-order advanced difference equation

$$
\Delta y(t)=\theta_{0} \Lambda_{0}(t) y^{\frac{\alpha_{3}}{\alpha_{1}}}\left(\xi_{0}(t)\right)
$$

and the first-order delay difference equations

$$
\begin{aligned}
& \Delta Z(t)+\theta_{1} \Lambda_{1}(t) Z^{\frac{\alpha_{2}}{\alpha_{1}}}\left(\xi_{1}(t)\right)=0, \\
& \Delta Z(t)+\Lambda_{2}(t) Z^{\frac{\alpha_{2}}{\alpha_{1} \alpha_{5}}}\left(\xi_{2}(t)\right)=0,
\end{aligned}
$$

and

$$
\Delta Z(t)+\Lambda_{3}(t) Z^{\frac{\alpha_{2}}{\alpha_{1} \alpha_{5}}}\left(\xi_{3}(t)\right)=0
$$

are oscillatory, then so is (1.1). 
Proof Assume that $x$ is a nonoscillatory solution of (1.1), say

$$
x(t)>0, \quad x(t-k)>0, \quad x(t-m+1)>0, \quad x\left(t+m^{*}\right)>0
$$

eventually. It follows from (1.1) that, eventually,

$$
\Delta\left(p_{1}(t)\left(\Delta^{2} y(t)\right)^{\alpha_{1}}\right)=p_{2}(t) x^{\alpha_{2}}(t-m+1)+p_{3}(t) x^{\alpha_{3}}\left(t+m^{*}\right)>0 .
$$

Hence (2.3) is satisfied, and thus, by Lemma 2.3, only the four Cases PPP, PPN, NNN, and NPN are possible. We now discuss each of these possible cases.

Cases PPP and PPN. Applying (2.1) with

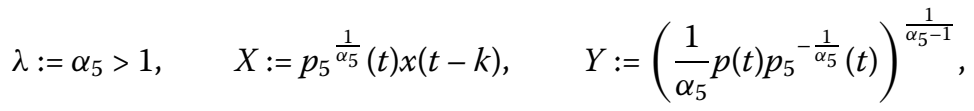

we obtain

$$
p(t) x(t-k)-p_{5}(t) x^{\alpha_{5}}(t-k) \leq g_{2}(t),
$$

while applying (2.2) with

$$
\lambda:=\alpha_{4} \in(0,1), \quad X:=p_{4}^{\frac{1}{\alpha_{4}}}(t) x(t-k), \quad Y:=\left(\frac{1}{\alpha_{4}} p(t) p_{4}^{-\frac{1}{\alpha_{4}}}(t)\right)^{\frac{1}{\alpha_{4}-1}}
$$

we get

$$
-\left(p(t) x(t-k)-p_{4}(t) x^{\alpha_{4}}(t-k)\right) \leq g_{1}(t) .
$$

Using these two inequalities, we have

$$
\begin{aligned}
x(t) & =y(t)-\left(p(t) x(t-k)-p_{5}(t) x^{\alpha_{5}}(t-k)\right)+\left(p(t) x(t-k)-p_{4}(t) x^{\alpha_{4}}(t-k)\right) \\
& \geq y(t)-g_{1}(t)-g_{2}(t)=\left[1-\frac{g_{1}(t)+g_{2}(t)}{y(t)}\right] y(t) .
\end{aligned}
$$

Since $y$ in both Cases PPP and PPN is positive and nondecreasing, there exists $C>0$ satisfying $y(t) \geq C$, and so we have

$$
x(t) \geq\left[1-\frac{g_{1}(t)+g_{2}(t)}{C}\right] y(t) .
$$

Next, due to (3.1), there exists $\kappa \in(0,1)$ such that

$$
x(t) \geq \kappa y(t) \quad \text { eventually. }
$$

Thus, we have

$$
\Delta\left(p_{1}(t)\left(\Delta^{2} y(t)\right)^{\alpha_{1}}\right) \geq \kappa^{\alpha_{2}} p_{2}(t) y^{\alpha_{2}}(t-m+1)+\kappa^{\alpha_{3}} p_{3}(t) y^{\alpha_{3}}\left(t+m^{*}\right) \geq 0 .
$$


Case PPP. By (3.8), we get

$$
\Delta\left(p_{1}(t)\left(\Delta^{2} y(t)\right)^{\alpha_{1}}\right) \geq \kappa^{\alpha_{3}} p_{3}(t) y^{\alpha_{3}}\left(t+m^{*}\right) .
$$

Summing (3.9) from $t-k_{0}$ to $t-1$, we get

$$
\begin{aligned}
p_{1}(t)\left(\Delta^{2} y(t)\right)^{\alpha_{1}} & =p_{1}\left(t-k_{0}\right)\left(\Delta^{2} y\left(t-k_{0}\right)\right)^{\alpha_{1}}+\sum_{\tau=t-k_{0}}^{t-1} \Delta\left(p_{1}(\tau)\left(\Delta^{2} y(\tau)\right)^{\alpha_{1}}\right) \\
& \stackrel{(3.9)}{\geq} \kappa^{\alpha_{3}} \sum_{\tau=t-k_{0}}^{t-1} p_{3}(\tau) y^{\alpha_{3}}\left(\tau+m^{*}\right) \\
& \geq \kappa^{\alpha_{3}} y^{\alpha_{3}}\left(t+m^{*}-k_{0}\right) \sum_{\tau=t-k_{0}}^{t-1} p_{3}(\tau) .
\end{aligned}
$$

Therefore, we have

$$
\Delta^{2} y(t) \geq \kappa^{\frac{\alpha_{3}}{\alpha_{1}}} y^{\frac{\alpha_{3}}{\alpha_{1}}}\left(t+m^{*}-k_{0}\right)\left(\frac{1}{p_{1}(t)} \sum_{\tau=t-k_{0}}^{t-1} p_{3}(\tau)\right)^{\frac{1}{\alpha_{1}}}
$$

Summing (3.10) again from $t-k_{0}$ to $t-1$, we obtain

$$
\begin{aligned}
\Delta y(t) & =\Delta y\left(t-k_{0}\right)+\sum_{\tau=t-k_{0}}^{t-1} \Delta^{2} y(\tau) \\
& \stackrel{(3.10)}{\geq} \kappa^{\frac{\alpha_{3}}{\alpha_{1}}} \sum_{\tau=t-k_{0}}^{t-1} y^{\frac{\alpha_{3}}{\alpha_{1}}}\left(\tau+m^{*}-k_{0}\right)\left(\frac{1}{p_{1}(\tau)} \sum_{s=\tau-k_{0}}^{\tau-1} p_{3}(s)\right)^{\frac{1}{\alpha_{1}}} \\
& \geq \kappa^{\frac{\alpha_{3}}{\alpha_{1}}} y^{\frac{\alpha_{3}}{\alpha_{1}}}\left(t+m^{*}-2 k_{0}\right) \sum_{\tau=t-k_{0}}^{t-1}\left(\frac{1}{p_{1}(\tau)} \sum_{s=\tau-k_{0}}^{\tau-1} p_{3}(s)\right)^{\frac{1}{\alpha_{1}}} .
\end{aligned}
$$

In summary, $y$ is a positive and increasing solution of

$$
\Delta y(t)-\kappa^{\frac{\alpha_{3}}{\alpha_{1}}} \Lambda_{0}(t) y^{\frac{\alpha_{3}}{\alpha_{1}}}\left(\xi_{0}(t)\right) \geq 0 .
$$

Employing Lemma 2.1 (II), (3.2) also has an eventually positive solution, which is a contradiction.

Case PPN. We introduce

$$
Z:=-p_{1}\left(\Delta^{2} y\right)^{\alpha_{1}}>0 \quad \text { eventually. }
$$

By (3.8), we obtain

$$
-\Delta Z(t) \geq \kappa^{\alpha_{2}} p_{2}(t) y^{\alpha_{2}}(t-m+1) .
$$


First, we see that, eventually,

$$
\begin{aligned}
y(t) & =y\left(t_{1}\right)+\sum_{\tau=t_{1}}^{t-1} \Delta y(\tau) \geq \sum_{\tau=t_{1}}^{t-1} \Delta y(\tau) \\
& \geq \sum_{\tau=t_{1}}^{t-1} \Delta y(t-1)=\left(t-t_{1}\right) \Delta y(t-1)=t \Delta y(t-1)\left(1-\frac{t_{1}}{t}\right) .
\end{aligned}
$$

Since $t_{1} / t \rightarrow 0$ as $t \rightarrow \infty$, there exists $\theta \in(0,1)$ so that

$$
y(t) \geq \theta t \Delta y(t-1) \quad \text { eventually. }
$$

Now, we put

$$
u:=t-m \quad \text { and } \quad v:=u+k_{1}>u .
$$

Then, eventually,

$$
\begin{aligned}
& 0 \leq \Delta y(v)=\Delta y(u)+\sum_{\tau=u}^{v-1} \Delta^{2} y(\tau) \\
& \stackrel{(3.11)}{=} \Delta y(u)-\sum_{\tau=u}^{v-1} \frac{Z^{\frac{1}{\alpha_{1}}}(\tau)}{p_{1}^{\frac{1}{\alpha_{1}}}(\tau)} \\
& \stackrel{(3.6)}{\leq} \Delta y(u)-\sum_{\tau=u}^{v-1} \frac{Z^{\frac{1}{\alpha_{1}}}(v-1)}{p_{1}^{\frac{1}{\alpha_{1}}}(\tau)} \\
&=\Delta y(u)-Z^{\frac{1}{\alpha_{1}}}(v-1) P_{1}(v, u),
\end{aligned}
$$

and hence

$$
\Delta y(u) \geq Z^{\frac{1}{\alpha_{1}}}(v-1) P_{1}(v, u) \quad \text { eventually. }
$$

Altogether, eventually,

$$
\begin{aligned}
-\Delta Z(t) & \stackrel{(3.12)}{\geq} \kappa^{\alpha_{2}} p_{2}(t) y^{\alpha_{2}}(t-m+1) \\
& \stackrel{(3.13)}{\geq}(\theta \kappa)^{\alpha_{2}} p_{2}(t)(t-m+1)^{\alpha_{2}}(\Delta y(t-m))^{\alpha_{2}} \\
& =(\theta \kappa)^{\alpha_{2}} p_{2}(t)(t-m+1)^{\alpha_{2}}(\Delta y(u))^{\alpha_{2}} \\
& \stackrel{(3.14)}{\geq}(\theta \kappa)^{\alpha_{2}} p_{2}(t)(t-m+1)^{\alpha_{2}}\left(Z^{\frac{1}{\alpha_{1}}}(v-1) P_{1}(v, u)\right)^{\alpha_{2}} \\
& =(\theta \kappa)^{\alpha_{2}} p_{2}(t)(t-m+1)^{\alpha_{2}}\left(Z^{\frac{1}{\alpha_{1}}}\left(\xi_{1}(t)\right) P_{1}\left(t-m+k_{1}, t-m\right)\right)^{\alpha_{2}} .
\end{aligned}
$$

In summary, $Z$ is a positive and decreasing solution of

$$
\Delta Z(t)+(\theta \kappa)^{\alpha_{2}} \Lambda_{1}(t) Z^{\frac{1}{\alpha_{1}}}\left(\xi_{1}(t)\right) \leq 0
$$


Employing Lemma 2.1 (I), (3.3) also has an eventually positive solution, which is a contradiction.

Cases NNN and NPN. Throughout the remainder of the proof, we introduce $Z$ again by (3.11). First note that, eventually,

$$
y(t)=x(t)+p_{4}(t) x^{\alpha_{4}}(t-k)-p_{5}(t) x^{\alpha_{5}}(t-k) \geq-p_{5}(t) x^{\alpha_{5}}(t-k) .
$$

Hence, eventually,

$$
x(t-k) \geq-\left(\frac{y(t)}{p_{5}(t)}\right)^{\frac{1}{\alpha_{5}}} .
$$

Thus, eventually,

$$
\begin{aligned}
-\Delta Z(t) & \stackrel{(1.1)}{=} p_{2}(t) x^{\alpha_{2}}(t-m+1)+p_{3}(t) x^{\alpha_{5}}\left(t+m^{*}\right) \\
& \geq p_{2}(t) x^{\alpha_{2}}(t-m+1) \stackrel{(3.15)}{\geq}-p_{2}(t)\left(\frac{y(t-m+k+1)}{p_{5}(t-m+k+1)}\right)^{\frac{\alpha_{2}}{\alpha_{5}}} \\
& =-Q(t) y^{\frac{\alpha_{2}}{\alpha_{5}}}(t-m+k+1) .
\end{aligned}
$$

Case NNN. First note that, eventually,

$$
\begin{aligned}
\Delta y(t) & =\Delta y\left(t_{1}\right)+\sum_{\tau=t_{1}}^{t-1} \Delta^{2} y(\tau) \leq \sum_{\tau=t_{1}}^{t-1} \Delta^{2} y(\tau) \\
& \stackrel{(3.11)}{=}-\sum_{\tau=t_{1}}^{t-1} \frac{Z^{\frac{1}{\alpha_{1}}}(\tau)}{p_{1}^{\frac{1}{\alpha_{1}}}(\tau)} \\
& \stackrel{(3.6)}{\leq}-\sum_{\tau=t_{1}}^{t-1} \frac{Z^{\frac{1}{\alpha_{1}}}(t-1)}{p_{1}{ }^{\frac{1}{\alpha_{1}}}(\tau)} \\
= & -Z^{\frac{1}{\alpha_{1}}}(t-1) P_{1}\left(t, t_{1}\right)
\end{aligned}
$$

and therefore, eventually,

$$
\begin{aligned}
y(t) & =y\left(t_{1}\right)+\sum_{\tau=t_{1}}^{t-1} \Delta y(\tau) \\
& \leq \sum_{\tau=t_{1}}^{t-1} \Delta y(\tau) \leq-\sum_{\tau=t_{1}}^{t-1} Z^{\frac{1}{\alpha_{1}}}(\tau-1) P_{1}\left(\tau, t_{1}\right) \\
& \stackrel{(3.6)}{\leq}-Z^{\frac{1}{\alpha_{1}}}(t-2) \sum_{\tau=t_{1}}^{t-1} P_{1}\left(\tau, t_{1}\right),
\end{aligned}
$$


and thus, eventually,

$$
\begin{aligned}
-\Delta Z(t) & \stackrel{(3.16)}{\geq}-Q(t) y^{\frac{\alpha_{2}}{\alpha_{5}}}(t-m+k+1) \\
& \geq Q(t)\left(Z^{\frac{1}{\alpha_{1}}}(t-m+k-1) \sum_{\tau=t_{1}}^{t-m+k} P_{1}\left(\tau, t_{1}\right)\right)^{\frac{\alpha_{2}}{\alpha_{5}}} \\
& =Q(t)\left(\sum_{\tau=t_{1}}^{t-m+k} P_{1}\left(\tau, t_{1}\right)\right)^{\frac{\alpha_{2}}{\alpha_{5}}} Z^{\frac{\alpha_{2}}{\alpha_{1} \alpha_{5}}}\left(\xi_{2}(t)\right) .
\end{aligned}
$$

In summary, $Z$ is a positive and decreasing solution of

$$
\Delta Z(t)+\Lambda_{2}(t) Z^{\frac{\alpha_{2}}{\alpha_{1} \alpha_{5}}}\left(\xi_{2}(t)\right) \leq 0 .
$$

Employing Lemma 2.1 (I), (3.4) also has an eventually positive solution, which is a contradiction.

Case NPN. We let

$$
u:=t-m+k+1, \quad v:=u+k_{2}>u, \quad \text { and } \quad w:=u+k_{3}-1>v-1 .
$$

First, we have, eventually,

$$
\begin{aligned}
& 0 \geq y(v)=y(u)+\sum_{\tau=u}^{v-1} \Delta y(\tau) \\
& \geq y(u)+\sum_{\tau=u}^{v-1} \Delta y(v-1)=y(u)+(v-u) \Delta y(v-1),
\end{aligned}
$$

so,

$$
-y(u) \geq(v-u) \Delta y(v-1) .
$$

Next, we have, eventually,

$$
\begin{aligned}
0 & \leq \Delta y(w)=\Delta y(v-1)+\sum_{\tau=v-1}^{w-1} \Delta^{2} y(\tau) \\
& \stackrel{(3.11)}{=} \Delta y(v-1)-\sum_{\tau=v-1}^{w-1} \frac{Z^{\frac{1}{\alpha_{1}}}(\tau)}{p_{1}^{\frac{1}{\alpha_{1}}}(\tau)} \\
& \stackrel{(3.6)}{\leq} \Delta y(v-1)-\sum_{\tau=v-1}^{w-1} \frac{Z^{\frac{1}{\alpha_{1}}}(w-1)}{p^{\frac{1}{\alpha_{1}}}(\tau)} \\
& =\Delta y(v-1)-Z^{\frac{1}{\alpha_{1}}}(w-1) P_{1}(w, v-1),
\end{aligned}
$$

so,

$$
\Delta y(v-1) \geq Z^{\frac{1}{\alpha_{1}}}(w-1) P_{1}(w, v-1) .
$$


Thus, we see that

$$
\begin{aligned}
-\Delta Z(t) & \stackrel{(3.16)}{\geq}-Q(t) y^{\frac{\alpha_{2}}{\alpha_{5}}}(t-m+k+1)=-Q(t) y^{\frac{\alpha_{2}}{\alpha_{5}}}(u) \\
& \stackrel{(3.17)}{\geq} Q(t)((v-u) \Delta y(v-1))^{\frac{\alpha_{2}}{\alpha_{5}}}=Q(t) k_{2}^{\frac{\alpha_{2}}{\alpha_{5}}}(\Delta y(v-1))^{\frac{\alpha_{2}}{\alpha_{5}}} \\
& \stackrel{(3.18)}{\geq} Q(t) k_{2}^{\frac{\alpha_{2}}{\alpha_{5}}}\left(Z^{\frac{1}{\alpha_{1}}}(w-1) P_{1}(w, v-1)\right)^{\frac{\alpha_{2}}{\alpha_{5}}} \\
& =Q(t) k_{2}^{\frac{\alpha_{2}}{\alpha_{5}}} P_{1}^{\frac{\alpha_{2}}{\alpha_{5}}}\left(t-m+k+k_{3}, t-m+k+k_{2}\right) Z^{\frac{\alpha_{2}}{\alpha_{1} \alpha_{5}}}\left(\xi_{3}(t)\right) .
\end{aligned}
$$

In summary, $Z$ is a positive and decreasing solution of

$$
\Delta Z(t)+\Lambda_{3}(t) Z^{\frac{\alpha_{2}}{\alpha_{1} \alpha_{5}}}\left(\xi_{3}(t)\right) \leq 0
$$

Employing Lemma 2.1 (I), (3.5) also has an eventually positive solution, which is a contradiction.

We now prove the following consequence of Theorem 3.1.

Theorem 3.2 Let $\alpha_{4}<1<\alpha_{5}$. Suppose that (i)-(iii), (1.2), (2.17), and (3.1) hold. Let $\theta_{0}, \theta_{1} \in(0,1)$. If the first-order advanced difference equation (3.2) and the first-order delay difference equations (3.3) and

$$
\Delta Z(t)+\min \left\{\Lambda_{2}(t), \Lambda_{3}(t)\right\} Z^{\frac{\alpha_{2}}{\alpha_{1} \alpha_{5}}}\left(\xi_{3}(t)\right)=0
$$

are oscillatory, then so is (1.1).

Proof We claim that oscillation of (3.19) implies oscillation of both (3.4) and (3.5). As all the other assumptions are the same as in Theorem 3.1, the statement then follows from Theorem 3.1. So assume that (3.19) is oscillatory. First, suppose that (3.4) is not oscillatory, say, there exists eventually positive $Z$ satisfying

$$
0=\Delta Z(t)+\Lambda_{2}(t) Z^{\frac{\alpha_{2}}{\alpha_{1} \alpha_{5}}}\left(\xi_{2}(t)\right) \geq \Delta Z(t)+\min \left\{\Lambda_{2}(t), \Lambda_{3}(t)\right\} Z^{\frac{\alpha_{2}}{\alpha_{1} \alpha_{5}}}\left(\xi_{2}(t)\right) .
$$

From the equality in (3.20), we see that $Z$ is eventually decreasing, and since

$$
\xi_{3}(t)=\xi_{2}(t)+k_{3}>\xi_{2}(t)
$$

we obtain $Z\left(\xi_{3}(t)\right) \leq Z\left(\xi_{2}(t)\right)$ eventually. Using this in (3.20), we get

$$
0 \geq \Delta Z(t)+\min \left\{\Lambda_{2}(t), \Lambda_{3}(t)\right\} Z^{\frac{\alpha_{2}}{\alpha_{1} \alpha_{5}}}\left(\xi_{2}(t)\right) \geq \Delta Z(t)+\min \left\{\Lambda_{2}(t), \Lambda_{3}(t)\right\} Z^{\frac{\alpha_{2}}{\alpha_{1} \alpha_{5}}}\left(\xi_{3}(t)\right) .
$$

By Lemma 2.1 (I), (3.19) also has an eventually positive solution, a contradiction, showing that (3.4) is indeed oscillatory. Next, suppose that (3.5) is not oscillatory, say, there exists eventually positive $Z$ satisfying

$$
0=\Delta Z(t)+\Lambda_{3}(t) Z^{\frac{\alpha_{2}}{\alpha_{1} \alpha_{5}}}\left(\xi_{3}(t)\right) \geq \Delta Z(t)+\min \left\{\Lambda_{2}(t), \Lambda_{3}(t)\right\} Z^{\frac{\alpha_{2}}{\alpha_{1} \alpha_{5}}}\left(\xi_{3}(t)\right) .
$$


By Lemma 2.1 (I), (3.19) also has an eventually positive solution, a contradiction, showing that (3.5) is indeed oscillatory as well. Thus, the proof is complete.

Remark 3.3 Observe that the minimum occurring in (3.19) may be calculated as follows:

$$
\min \left\{\Lambda_{2}(t), \Lambda_{3}(t)\right\}=Q(t)\left(\min \left\{\sum_{\tau=t_{1}}^{t-m+k} P_{1}\left(\tau, t_{1}\right), k_{2} P_{1}\left(t-m+k+k_{3}, t-m+k+k_{2}\right)\right\}\right)^{\frac{\alpha_{2}}{\alpha_{5}}} .
$$

The following theorem gives some further criteria for a special case.

Theorem 3.4 Let $\alpha_{4}<1<\alpha_{5}$ and $\alpha_{2} \leq \alpha_{1} \leq \alpha_{3}$. Suppose that (i)-(iii), (1.2), (2.17), and (3.1) hold. If

$$
\begin{aligned}
& \limsup _{t \rightarrow \infty} \sum_{\tau=t}^{\xi_{0}(t)-1} \Lambda_{0}(\tau)=\infty, \\
& \limsup _{t \rightarrow \infty} \sum_{\tau=\xi_{1}(t)}^{t} \Lambda_{1}(\tau)=\infty, \\
& \limsup _{t \rightarrow \infty} \sum_{\tau=\xi_{2}(t)}^{t} \Lambda_{2}(\tau)=\infty,
\end{aligned}
$$

and

$$
\limsup _{t \rightarrow \infty} \sum_{\tau=\xi_{3}(t)}^{t} \Lambda_{3}(\tau)=\infty
$$

then (1.1) is oscillatory.

Proof We claim that under the additional assumption $\alpha_{2} \leq \alpha_{1} \leq \alpha_{3}$, (3.21), (3.22), (3.23), and (3.24) imply oscillation of (3.2), (3.3), (3.4), and (3.5), respectively. As all the other assumptions are the same as in Theorem 3.1, the statement then follows from Theorem 3.1. First, suppose that (3.2) is not oscillatory, say, there exists eventually positive $y$ satisfying (3.2). As can be seen from (3.2), $y$ is eventually increasing and thus bounded below by some $C>0$. Summing (3.2) from $\tau=t$ to $\tau=\xi_{0}(t)-1$, we obtain, eventually,

$$
y\left(\xi_{0}(t)\right) \geq y\left(\xi_{0}(t)\right)-y(t) \stackrel{(3.2)}{=} \sum_{\tau=t}^{\xi_{0}(t)-1} \theta_{0} \Lambda_{0}(\tau) y^{\frac{\alpha_{3}}{\alpha_{1}}}\left(\xi_{0}(\tau)\right) \geq \sum_{\tau=t}^{\xi_{0}(t)-1} \theta_{0} \Lambda_{0}(\tau) y^{\frac{\alpha_{3}}{\alpha_{1}}}\left(\xi_{0}(t)\right),
$$

and thus, as $\alpha_{3} \geq \alpha_{1}$,

$$
\theta_{0} \sum_{\tau=t}^{\xi_{0}(t)-1} \Lambda_{0}(\tau) \leq \frac{1}{y^{\frac{\alpha_{3}-\alpha_{1}}{\alpha_{1}}}\left(\xi_{0}(t)\right)} \leq \frac{1}{C^{\frac{\alpha_{3}-\alpha_{1}}{\alpha_{1}}}}
$$

contradicting (3.21). Next, suppose that (3.3) is not oscillatory, say, there exists eventually positive $Z$ satisfying (3.3). As can be seen from (3.3), $Z$ is eventually decreasing and thus 
bounded above by some $C>0$. Summing (3.3) from $\tau=\xi_{1}(t)$ to $\tau=t$, we obtain, eventually,

$$
Z\left(\xi_{1}(t)\right) \geq Z\left(\xi_{1}(t)\right)-Z(t+1) \stackrel{(3.3)}{=} \sum_{\tau=\xi_{1}(t)}^{t} \theta_{1} \Lambda_{1}(\tau) Z^{\frac{\alpha_{2}}{\alpha_{1}}}\left(\xi_{1}(t)\right),
$$

and thus, as $\alpha_{1} \geq \alpha_{2}$,

$$
\theta_{1} \sum_{\tau=\xi_{1}(t)}^{t} \Lambda_{1}(\tau) \leq Z^{\frac{\alpha_{1}-\alpha_{2}}{\alpha_{1}}}\left(\xi_{1}(t)\right) \leq C^{\frac{\alpha_{1}-\alpha_{2}}{\alpha_{1}}}
$$

contradicting (3.22). Next, suppose that (3.4) is not oscillatory, say, there exists eventually positive $Z$ satisfying (3.4). As can be seen from (3.4), $Z$ is eventually decreasing and thus bounded above by some $C>0$. Summing (3.4) from $\tau=\xi_{2}(t)$ to $\tau=t$, we obtain, eventually,

$$
\begin{aligned}
Z\left(\xi_{2}(t)\right) & \geq Z\left(\xi_{2}(t)\right)-Z(t+1) \stackrel{(3.4)}{=} \sum_{\tau=\xi_{2}(t)}^{t} \Lambda_{2}(\tau) Z^{\frac{\alpha_{2}}{\alpha_{1} \alpha_{5}}}\left(\xi_{2}(\tau)\right) \\
& \geq \sum_{\tau=\xi_{2}(t)}^{t} \Lambda_{2}(\tau) Z^{\frac{\alpha_{2}}{\alpha_{1} \alpha_{5}}}\left(\xi_{2}(t)\right),
\end{aligned}
$$

and thus, as $\alpha_{1} \alpha_{5} \geq \alpha_{1} \geq \alpha_{2}$,

$$
\sum_{\tau=\xi_{2}(t)}^{t} \Lambda_{2}(\tau) \leq Z^{\frac{\alpha_{1} \alpha_{5}-\alpha_{2}}{\alpha_{1} \alpha_{5}}}\left(\xi_{2}(t)\right) \leq C^{\frac{\alpha_{1} \alpha_{5}-\alpha_{2}}{\alpha_{1} \alpha_{5}}}
$$

contradicting (3.23). The proof that (3.24) implies oscillation of (3.5) follows exactly like the proof that (3.23) implies oscillation of (3.4), only with $\xi_{2}$ and $\Lambda_{2}$ replaced by $\xi_{3}$ and $\Lambda_{3}$, respectively.

Remark 3.5 Note that Tang and Liu [34] have developed other oscillation criteria for sublinear delay difference equations, which could be used in place of (3.22), (3.23), and (3.24) to examine the oscillation of (3.3), (3.4), and (3.5). Similar criteria for superlinear advanced difference equations could not be found in the literature.

Now, we focus on the case $\alpha_{4}<\alpha_{5} \leq 1$.

Theorem 3.6 Let $\alpha_{4}<\alpha_{5} \leq 1$. Suppose that (i)-(iii), (1.2), and (2.17) hold. Assume

$$
\lim _{t \rightarrow \infty} P(t)=0, \quad \text { where } P(t):=\frac{\alpha_{5}-\alpha_{4}}{\alpha_{4}}\left(\frac{\alpha_{4}}{\alpha_{5}} p_{4}(t)\right)^{\frac{\alpha_{5}}{\alpha_{5}-\alpha_{4}}}\left(p_{5}(t)\right)^{\frac{\alpha_{4}}{\alpha_{4}-\alpha_{5}}}
$$

Let $\theta_{0}, \theta_{1} \in(0,1)$. If (3.2), (3.3), (3.4), and (3.5) are oscillatory, then so is (1.1).

Proof Inspecting the proof of Theorem 3.1, we see that $\alpha_{5}>1$ was needed only in the discussions under the headline "Cases PPP and PPN" leading to (3.7). The rest of the proof does not use $\alpha_{5}>1$ and remains unaffected. Thus, while we used (3.1) in Theorem 3.1 to 
show (3.7), we will now use (3.25) to show (3.7), hence completing the proof. Applying (2.1) with

$$
\lambda:=\frac{\alpha_{5}}{\alpha_{4}}>1, \quad X:=x^{\alpha_{4}}(t-k), \quad Y:=\left(\frac{\alpha_{4} p_{4}(t)}{\alpha_{5} p_{5}(t)}\right)^{\frac{\alpha_{4}}{\alpha_{5}-\alpha_{4}}}
$$

we obtain

$$
x^{\alpha_{5}}(t-k)+\frac{\alpha_{5}-\alpha_{4}}{\alpha_{4}}\left(\frac{\alpha_{4} p_{4}(t)}{\alpha_{5} p_{5}(t)}\right)^{\frac{\alpha_{5}}{\alpha_{5}-\alpha_{4}}} \geq \frac{\alpha_{5}}{\alpha_{4}} x^{\alpha_{4}}(t-k) \frac{\alpha_{4} p_{4}(t)}{\alpha_{5} p_{5}(t)},
$$

and thus

$$
\begin{aligned}
x(t) & =y(t)-\left(p_{4}(t) x^{\alpha_{4}}(t-k)-p_{5}(t) x^{\alpha_{5}}(t-k)\right) \\
& \geq y(t)-P(t)=\left(1-\frac{P(t)}{y(t)}\right) y(t) .
\end{aligned}
$$

Since $y$ is positive and nondecreasing, there exists $C>0$ such that $y(t) \geq C$, and so we have

$$
x(t) \geq\left(1-\frac{P(t)}{C}\right) y(t)
$$

Next, due to (3.25), there exists $\kappa \in(0,1)$ such that (3.7) is satisfied. This completes the proof.

As before in Theorem 3.2 and Theorem 3.4, we now obtain the following results.

Theorem 3.7 Let $\alpha_{4}<\alpha_{5} \leq 1$. Suppose that (i)-(iii), (1.2), (2.17), and (3.25) hold. Let $\theta_{0}, \theta_{1} \in(0,1)$. If (3.2), (3.3), and (3.19) are oscillatory, then so is (1.1).

Theorem 3.8 Let $\alpha_{4}<\alpha_{5} \leq 1$ and $\alpha_{2} / \alpha_{5} \leq \alpha_{1} \leq \alpha_{3}$. Suppose that (i)-(iii), (1.2), (2.17), and (3.25) hold. If (3.21), (3.22), (3.23), and (3.24) hold, then (1.1) is oscillatory.

\section{Examples}

We conclude this paper by giving two examples, illustrating our theoretical findings.

Example 4.1 We consider the equation

$$
\begin{aligned}
& \Delta\left((t+1)^{3}\left(\Delta^{2}\left(x(t)+\frac{1}{t} x^{\frac{1}{3}}(t-1)-x^{3}(t-1)\right)\right)^{3}\right) \\
& \quad=t^{2} x(t-2)+(t+2)^{4} x(t+6) .
\end{aligned}
$$

Then (4.1) is in the form (1.1), where

$$
\begin{array}{lll}
\alpha_{1}=\alpha_{3}=\alpha_{5}=3, & \alpha_{2}=1, \quad \alpha_{4}=\frac{1}{3}, \quad k=1, m=3, m^{*}=6, \\
p_{1}(t)=(t+1)^{3}, \quad p_{2}(t)=t^{2}, & p_{3}(t)=(t+2)^{4}, \quad p_{4}(t)=\frac{1}{t}, \quad p_{5}(t)=1 .
\end{array}
$$


Next, (i)-(iii) are satisfied, and so is (1.2) due to

$$
P_{1}(\nu, u)=\sum_{\tau=u}^{v-1}\left(\frac{1}{p_{1}(\tau)}\right)^{\frac{1}{3}}=\sum_{\tau=u}^{\nu-1} \frac{1}{\tau+1}=\sum_{\tau=u+1}^{v} \frac{1}{\tau} \rightarrow \infty
$$

Now we may pick (see Remark 2.4)

$$
k_{0}=k_{1}=k_{2}=1 \quad \text { and } \quad k_{3}=2 \text {, }
$$

and then (2.17) is satisfied, and we have

$$
\xi_{0}(t)=t+4, \quad \xi_{1}(t)=\xi_{2}(t)=t-3, \quad \xi_{3}(t)=t-1 .
$$

Moreover, we have $\alpha_{4}<1<\alpha_{5}$ and $\alpha_{2}<\alpha_{1}=\alpha_{3}$, so we will apply Theorem 3.4. We pick $p=p_{4}$, and then

$$
\begin{aligned}
g_{1}(t)+g_{2}(t) & =\frac{2}{3}\left(\frac{1}{3}\right)^{\frac{1}{2}}(p(t))^{-\frac{1}{2}}\left(p_{4}(t)\right)^{\frac{3}{2}}+2 \cdot 3^{-\frac{3}{2}}(p(t))^{\frac{3}{2}}\left(p_{5}(t)\right)^{-\frac{1}{2}} \\
& =\frac{2}{3 \sqrt{3} t}\left(1+\frac{1}{\sqrt{t}}\right) \rightarrow 0 \quad \text { as } t \rightarrow \infty,
\end{aligned}
$$

and thus, (3.1) is satisfied. We also calculate

$$
\begin{aligned}
& \Lambda_{0}(t)=\sum_{\tau=t-1}^{t-1}\left(\frac{1}{p_{1}(\tau)} \sum_{s=\tau-1}^{\tau-1} p_{3}(s)\right)^{\frac{1}{3}}=\left(\frac{p_{3}(t-2)}{p_{1}(t-1)}\right)^{\frac{1}{3}}=\left(\frac{t^{4}}{t^{3}}\right)^{\frac{1}{3}}=t^{\frac{1}{3}}, \\
& \Lambda_{1}(t)=p_{2}(t)(t-2) P_{1}(t-2, t-3)=p_{2}(t)=t^{2}, \\
& \Lambda_{2}(t)=p_{2}(t) \sum_{\tau=t_{1}}^{t-2} P_{1}\left(\tau, t_{1}\right) \geq \frac{t^{2}}{t-2} \geq t
\end{aligned}
$$

and

$$
\Lambda_{3}(t)=p_{2}(t)\left(P_{1}(t, t-1)\right)^{\frac{1}{3}}=p_{2}(t)\left(\frac{1}{t}\right)^{\frac{1}{3}}=t^{\frac{5}{3}}
$$

Hence, (3.21), (3.22), (3.23), and (3.24) hold. Now all the conditions of Theorem 3.4 are fulfilled, and thus, (4.1) is oscillatory.

Example 4.2 We consider the equation

$$
\begin{aligned}
& \Delta\left((t+1)^{3}\left(\Delta^{2}\left(x(t)+\frac{1}{t} x^{\frac{1}{3}}(t-1)-x^{\frac{2}{3}}(t-1)\right)\right)^{3}\right) \\
& \quad=t^{2} x(t-2)+(t+2)^{4} x(t+6) .
\end{aligned}
$$

Note that all data in (4.2) are the same as in (4.1), except

$$
\alpha_{5}=\frac{2}{3}
$$


Moreover, we have $\alpha_{4}<\alpha_{5}<1$ and $\alpha_{2} / \alpha_{5}<\alpha_{1}=\alpha_{3}$, so we will apply Theorem 3.8. We calculate

$$
P(t)=\left(\frac{1}{2} p_{4}(t)\right)^{2}\left(p_{5}(t)\right)^{-1}=\frac{1}{4 t^{2}} \rightarrow 0 \quad \text { as } t \rightarrow \infty,
$$

and thus, (3.25) is satisfied. The fulfillment of all other conditions of Theorem 3.8 follows in the same way as in Example 4.1, and hence (4.2) is oscillatory.

Remark 4.3 The results of this paper may be extended to higher-order difference equations of the form

$$
\Delta\left(p_{1}(t)\left(\Delta^{n-1} y(t)\right)^{\alpha_{1}}\right)=p_{2}(t) x^{\alpha_{2}}(t-m+1)+p_{3}(t) x^{\alpha_{3}}\left(t+m^{*}\right)
$$

where $y(t)=x(t)+p_{4}(t) x^{\alpha_{4}}(t-k)-p_{5} x^{\alpha_{5}}(t-k)$. We leave the details for future consideration.

\section{Acknowledgements}

J. Alzabut would like to thank Prince Sultan University for supporting this work.

\section{Funding}

Not applicable.

\section{Availability of data and materials}

Not applicable.

\section{Competing interests}

The authors declare that they have no competing interests.

\section{Authors' contributions}

The authors have contributed equally and significantly to the contents of this paper. All authors read and approved the final manuscript.

\section{Author details}

${ }^{1}$ Department of Mathematics and General Sciences, Prince Sultan University, 11586 Riyadh, Saudi Arabia. ${ }^{2}$ Department of Mathematics and Statistics, Missouri S\&T, Rolla, Missouri 65409, USA. ${ }^{3}$ Department of Engineering Mathematics, Faculty of Engineering, Cairo University, Giza 12221, Egypt.

\section{Publisher's Note}

Springer Nature remains neutral with regard to jurisdictional claims in published maps and institutional affiliations.

Received: 7 August 2020 Accepted: 1 December 2020 Published online: 05 January 2021

\section{References}

1. Agarwal, R.P.: Difference Equations and Inequalities: Theory, Methods, and Applications, 2nd edn. Monographs and Textbooks in Pure and Applied Mathematics, vol. 228. Dekker, New York (2000)

2. Agarwal, R.P., Bohner, M., Grace, S.R., O'Regan, D.: Discrete Oscillation Theory. Hindawi Publishing Corporation, New York (2005)

3. Agarwal, R.P., Bohner, M., Li, T., Zhang, C.: Hille and Nehari type criteria for third-order delay dynamic equations. J. Differ. Equ. Appl. 19(10), 1563-1579 (2013)

4. Agarwal, R.P., Bohner, M., Li, T., Zhang, C.: Oscillation of third-order nonlinear delay differential equations. Taiwan. J. Math. 17(2), 545-558 (2013)

5. Agarwal, R.P., Bohner, M., Li, T., Zhang, C.: Oscillation of second-order differential equations with a sublinear neutral term. Carpath. J. Math. 30(1), 1-6 (2014)

6. Agarwal, R.P., Bohner, M., Li, T., Zhang, C.: A philos-type theorem for third-order nonlinear retarded dynamic equations. Appl. Math. Comput. 249, 527-531 (2014)

7. Agarwal, R.P., Bohner, M., Tang, S., Li, T., Zhang, C.: Oscillation and asymptotic behavior of third-order nonlinear retarded dynamic equations. Appl. Math. Comput. 219(8), 3600-3609 (2012)

8. Agarwal, R.P., Grace, S.R., O'Regan, D.: Oscillation Theory for Difference and Functional Differential Equations. Kluwer Academic, Dordrecht (2000)

9. Aktaş, M.F., Tiryaki, A., Zafer, A.: Oscillation of third-order nonlinear delay difference equations. Turk. J. Math. 36(3), 422-436 (2012)

10. Bohner, M., Dharuman, C.P., Srinivasan, R., Thandapani, E.: Oscillation criteria for third-order nonlinear functional difference equations with damping. Appl. Math. Inf. Sci. 11(3), 669-676 (2017) 
11. Bohner, M., Geetha, S., Selvarangam, S., Thandapani, E.: Oscillation of third-order delay difference equations with negative damping term. Ann. Univ. Mariae Curie-Skłodowska, Sect. A 72(1), 19-28 (2018)

12. Bohner, M., Grace, S.R., Jadlovská, l.: Oscillation criteria for third-order functional differential equations with damping. Electron. J. Differ. Equ. 2016, 215 (2016)

13. Bohner, M., Grace, S.R., Jadlovská, I.: Asymptotic behavior of solutions of forced third-order dynamic equations. Analysis (Berlin) 39(1), 1-6 (2019)

14. Bohner, M., Grace, S.R., Sağer, I., Tunç, E.: Oscillation of third-order nonlinear damped delay differential equations. Appl. Math. Comput. 278, 21-32 (2016)

15. Bohner, M., Li, T.: Oscillation of second-order $p$-Laplace dynamic equations with a nonpositive neutral coefficient. Appl. Math. Lett. 37, 72-76 (2014)

16. Dharuman, C.P., Graef, J.R., Thandapani, E., Vidhyaa, K.S.: Oscillation of second order difference equation with a sub-linear neutral term. J. Math. Appl. 40, 59-67 (2017)

17. El-Morshedy, H.A.: Oscillation and nonoscillation criteria for half-linear second order difference equations. Dyn. Syst. Appl. 15(3-4), 429-450 (2006)

18. El-Morshedy, H.A.: New oscillation criteria for second order linear difference equations with positive and negative coefficients. Comput. Math. Appl. 58(10), 1988-1997 (2009)

19. El-Morshedy, H.A., Grace, S.R.: Comparison theorems for second order nonlinear difference equations. J. Math. Anal. Appl. 306(1), 106-121 (2005)

20. Grace, S.R.: Oscillatory behavior of third-order nonlinear difference equations with a nonlinear-nonpositive neutral term. Mediterr. J. Math. 16(5), Article ID 128 (2019)

21. Grace, S.R., Agarwal, R.P., Bohner, M., O'Regan, D.: Oscillation of second-order strongly superlinear and strongly sublinear dynamic equations. Commun. Nonlinear Sci. Numer. Simul. 14(8), 3463-3471 (2009)

22. Grace, S.R., Alzabut, J.: Oscillation results for nonlinear second order difference equations with mixed neutral terms. Adv. Differ. Equ. 2020, 8 (2020)

23. Grace, S.R., Bohner, M., Agarwal, R.P.: On the oscillation of second-order half-linear dynamic equations. J. Differ. Equ. Appl. 15(5), 451-460 (2009)

24. Grace, S.R., Bohner, M., Liu, A.: On Kneser solutions of third-order delay dynamic equations. Carpath. J. Math. 26(2), 184-192 (2010)

25. Grace, S.R., El-Morshedy, H.A.: Oscillation criteria of comparison type for second order difference equations. J. Appl. Anal. 6(1), 87-103 (2000)

26. Grace, S.R., Graef, J.R.: Oscillatory behavior of second order nonlinear differential equations with a sublinear neutral term. Math. Model. Anal. 23(2), 217-226 (2018)

27. Graef, J.R., Thandapani, E.: Oscillatory and asymptotic behavior of solutions of third order delay difference equations, Funkc. Ekvacioj 42(3), 355-369 (1999)

28. Hardy, G.H., Littlewood, J.E., Pólya, G.: Inequalities. Cambridge Mathematical Library. Cambridge University Press, Cambridge (1988) Reprint of the 1952 edition

29. Li, Q., Wang, C., Li, F., Liang, H., Zhang, Z.: Oscillation of sublinear difference equations with positive neutral term. J. Appl. Math. Comput. 20(1-2), 305-314 (2006)

30. Parhi, N.: Oscillations of first order difference equations. Proc. Indian Acad. Sci. Math. Sci. 110(2), 147-155 (2000)

31. Saker, S.H., Alzabut, J.O.: Oscillatory behavior of third order nonlinear difference equations with delayed argument. Dyn. Contin. Discrete Impuls. Syst., Ser. A Math. Anal. 17(5), 707-723 (2010)

32. Saker, S.H., Alzabut, J.O., Mukheimer, A.: On the oscillatory behavior for a certain class of third order nonlinear delay difference equations. Electron. J. Qual. Theory Differ. Equ. 2010, 67 (2010)

33. Shoukaku, Y:: On the oscillation of solutions of first-order difference equations with delay. Commun. Math. Anal. 20(2), 62-67 (2017)

34. Tang, X., Liu, Y.: Oscillation for nonlinear delay difference equations. Tamkang J. Math. 32(4), 275-280 (2001)

35. Thandapani, E., Selvarangam, S.: Oscillation results for third order half-linear neutral difference equations. Bull. Math. Anal. Appl. 4(2), 91-102 (2012)

36. Thandapani, E., Vijaya, M., Li, T.: On the oscillation of third order half-linear neutral type difference equations. Electron. J. Qual. Theory Differ. Equ. 2011, 76 (2011)

37. Yildiz, M.K., Öğünmez, H.: Oscillation results of higher order nonlinear neutral delay difference equations with a nonlinear neutral term. Hacet. J. Math. Stat. 43(5), 809-814 (2014)

\section{Submit your manuscript to a SpringerOpen ${ }^{\circ}$ journal and benefit from:}

- Convenient online submission

- Rigorous peer review

- Open access: articles freely available online

- High visibility within the field

- Retaining the copyright to your article

Submit your next manuscript at $\gg$ springeropen.com 